\title{
Cohen-Macaulay modules on hypersurface singularities II
}

\author{
R.-O. Buchweitz ${ }^{1, \star}$, G.-M. Greuel ${ }^{2}$, and F.-O. Schreyer ${ }^{2}$ \\ 1 Institut für Mathematik, Technische Universität Hannover, Welfengarten 1, D-3000 Hannover, \\ Federal Republic of Germany \\ 2 Fachbereich Mathematik, Universität Kaiserslautern. Erwin-Schrödınger-Str., \\ D-6750 Kaiserslautern, Federal Republic of Germany
}

Egbert Brieskorn gewidmet

\section{Contents}

0. Introduction and results . . . . . . . . . . . . . . . . . . . . . . . . . . . 165

1. Perjodic complexes and matrix factorizations . . . . . . . . . . . . . . . . . . . . . . . . . . . 169

2. Construction of matrix factorizations and MCM's . . . . . . . . . . . . . . . . . . . 173

3. Proof of the main results . . . . . . . . . . . . . . . . . . . . . . . . . . . . . 175

4. MCM's on $A_{x}$ and $D_{x}$. . . . . . . . . . . . . . . . . . . . . . . . . . . . 179

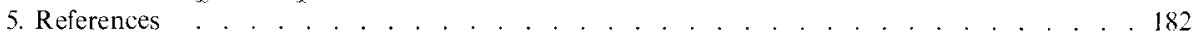

\section{Introduction and results}

0.1. H. Knörrer [Knö] showed the following property of the local ring $R$ of an isolated simple hypersurface singularity: There are only finitely many isomorphism classes of indecomposable maximal Cohen-Macaulay $R$-modules.

Recall that a module $M$ - always assumed to be finitely generated and unitary - over a local Cohen-Macaulay ring $R$ is said to be maximal CohenMacaulay, if depth $M=\operatorname{dim} R$ ( $M$ is an MCM for short). $M$ is called indecomposable if it is not a nontrivial direct sum. Let us call $R$ of finite (resp. countable) CM-representation type, if there are only finitely (resp. countably) many isomorphism classes of indecomposable MCM's over $R$. If $R$ is not of finite CM-representation type we say that it is of infinite CM-representation type.

The main object of this paper is to prove the converse of Knörrer's result, namely that a non-simple hypersurface singularity is of infinite CM-representation type (cf. Theorem A and B). This characterization of the simple hypersurface singularities has been proved before by Artin and Verdier [A-V], Auslander [Aus1] and Esnault [Esn] in Dimension 2 and by Greuel and Knörrer $[\mathrm{G}-\mathrm{K}]$ in Dimension 1.

In addition we classify the hypersurface singularities which are of countable CM-representation type (Theorem B). Our method yields also an application to

* Supported by a "Heisenberg-Stipendium", Bu-398/3-1 of the DFG 
vector bundles on projective hypersurfaces with "no cohomology in the middle" (Theorem C).

In our proof we explicitly construct infinitely many non isomorphic MCM's. The construction uses the fact that MCM's over a hypersurface ring $R$ have periodic resolutions and that every MCM arises from a matrix factorization of the defining function. This was first proved by D. Eisenbud [Eis] and we recall the main facts in $\S 1$.

0.2 . Let $P$ denote a regular noetherian local ring with maximal ideal $m$ and algebraically closed residue field $k=P / m$. Let $f \in m, f \neq 0, R=P /(f)$ and mult $R$ $=\operatorname{mult}(f)=\sup \left\{v \mid f \in m^{v}\right\}$.

We show in Proposition 3.1, that $R$ is of finite CM-representation type if either

a) $\operatorname{dim} R \geqq 1$ and mult $R \geqq 4$ or

b) $\operatorname{dim} R \geqq 2$ and mult $R \geqq 3$.

The modules which we construct are parametrized in Case a) by the points of $\mathbb{P}_{k}\left(m / m^{2}\right)$ and in Case b) by the points of a cubic in $\mathbb{P}_{k}\left(m / m^{2}\right)$. In order to arrive at the simple hypersurface singularities we specialize to analytic hypersurfaces. Suppose that $P$ is a regular analytic $k$-algebra, i.e. isomorphic to a formal or convergent (if $k$ is a complete non trivial valuated field) power series ring $k\left\langle z_{0}, \ldots, z_{n}\right\rangle . R=P /(f), f \in m$, is then called an analytic hypersurface ring and $(X, 0)$ denotes the analytic hypersurface defined by $R$.

Arnold introduced the notion of a simple hypersurface singularity over $k$ $=\mathbb{C}$ (or $\mathbb{R}$ ) for a singularity which is of "finite deformation type" and he classified them (cf. [Arn]). The classification yields the well-known singularities of type $A_{k}, D_{k}, E_{6}, E_{7}, E_{8}$. Barth, Peters and van de Ven [B-P-V] obtained for reduced plane curve singularities the same list from a different definition which has the advantage that it carries over immediately to arbitrary algebraically closed fields: A 1-dimensional reduced hypersurface ring $R=k\left\langle z_{0}, z_{1}\right\rangle /(f)$ is called simple if mult $(f) \leqq 3$ and if after any finitely many successive quadratic transformations, the reduced total transform of $f$ still has multiplicity $\leqq 3$ in each point. We then also call $f$ or $(X, 0)$ simple.

For the following theorem we assume that $R$ is an $n$-dimensional analytic hypersurface ring, $n \geqq 1, k$ algebraically closed and $\operatorname{char}(k) \neq 2$.

Theorem A. $R$ is of finite CM-representation type if and only if $R$ is isomorphic to $k\left\langle z_{0}, \ldots, z_{n}\right\rangle /(f)$ such that

$$
f\left(z_{0}, \ldots, z_{n}\right)=g\left(z_{0}, z_{1}\right)+z_{2}^{2}+\ldots+z_{n}^{2}
$$

where $g \in k\left\langle z_{0}, z_{1}\right\rangle$ is simple.

Remarks. 1) We need the assumption that $R$ is an analytic hypersurface ring in order to apply the Weierstraß preparation theorem and that $\operatorname{char}(k) \neq 2$ in order to split off $z_{2}^{2}+\ldots+z_{n}^{2}$. The assumption $\operatorname{char}(k) \neq 2$ is superfluous if $\operatorname{dim} R=1$ (cf. 3.5).

2) Equations of the simple singularities are given in [Arn], [B-P-V] and 0.3 for $k=\mathbb{C}$ and in $[\mathrm{K}-\mathrm{S}]$ for an arbitrary algebraically closed field. As was 
noticed in $[\mathrm{K}-\mathrm{S}]$, if $n=\operatorname{dim} R=2$ and $\operatorname{char}(k) \neq 2$, the singularities occurring in the theorem are exactly the rational double points in the sense of $[\mathrm{A}-\mathrm{V}]$.

3) As mentioned above, the theorem was proved before in Dimension 1 by [G-K] for $\operatorname{char}(k)=0$ and then extended to arbitrary characteristic by [K-S] (cf. also 3.5). In Dimension 2 it is due to [A-V], with different proofs also given in [Aus1] and [Esn]. Their result implies that a two dimensional normal analytic hypersurface singularity is of finite CM-representation type iff it is a rational double point, even in characteristic 2 .

0.3. In the following let $k=\mathbb{C}, f \in \mathbb{C}\left\{z_{0}, \ldots, z_{n}\right\}$ (the convergent power series ring), $R=\mathbb{C}\left\{z_{0}, \ldots, z_{n}\right\} /(f)$ and $(X, 0)$ the complex space germ defined by $R$. In this case, the singularities exhibited in Theorem B are exactly the simple singularities in the sense of Arnold, mentioned above:

$$
\begin{aligned}
& A_{k}: f=z_{0}^{k+1}+z_{1}^{2}+\ldots+z_{n}^{2}, \quad k \geqq 1, \\
& D_{k}: f=z_{0}^{k-1}+z_{0} z_{1}^{2}+z_{2}^{2}+\ldots+z_{n}^{2}, k \geqq 4, \\
& E_{6}: f=z_{0}^{3}+z_{1}^{4}+z_{2}^{2}+\ldots+z_{n}^{2}, \\
& E_{7}: f=z_{0}^{3}+z_{0} z_{1}^{3}+z_{2}^{2}+\ldots+z_{n}^{2}, \\
& E_{8}: f=z_{0}^{3}+z_{1}^{5}+z_{2}^{2}+\ldots+z_{n}^{2} .
\end{aligned}
$$

Notice that there are two natural limit cases of $A_{k}$ resp. $D_{k}$ as $k$ tends to infinity, namely

$$
\begin{aligned}
& A_{\infty}: f=z_{1}^{2}+\ldots+z_{n}^{2}, \\
& D_{\infty}: f=z_{0} z_{1}^{2}+z_{2}^{2}+\ldots+z_{n}^{2},
\end{aligned}
$$

considered as an element of $\mathbb{C}\left\{z_{0}, \ldots, z_{n}\right\}$.

Besides the simple ones, these two (non-isolated) singularities play a special role with respect to MCM's (cf. Theorem $\mathrm{B}$ ).

We mention also that $A_{\infty}$ and $D_{\infty}$ are basic for topological considerations in the deformation theory of non-isolated singularities (cf. [Sie]).

Let us call a singularity (i.e. a complex space germ) $(X, 0)$ of finite (resp. countable) deformation type, if there exist a finite (resp. countable) list of singularities with the following property: For any (flat) deformation $(Y, 0) \rightarrow(T, 0)$ of $(X, 0)$ over a complex space germ $(T, 0)$, there exists a representative $Y \rightarrow T$ such that each fibre $Y_{t}$ has only singularities which are isomorphic to one of the members of the list. A hypersurface singularity of finite deformation type is called simple. This is (a slight modification of) Arnold's definition and the classification leads to the singularities $A_{k}, D_{k}(k \neq \infty)$ and $E_{6}, E_{7}, E_{8}$.

Similarily we define an $R=\mathcal{O}_{X, 0^{-}}$-module $M$ to be of finite (resp. countable) deformation type if there exists a finite (resp. countable) list of $R$-modules such that for each deformation $\mathscr{A}$ of $M$ over $(T, 0)$ (i.e. $\mathscr{M}$ is a flat $\mathscr{C}_{X \times \boldsymbol{T}, 0}$-module and $M=\mathscr{M} \otimes \mathcal{O}_{X \times\{0\}, 0}$ ) there exists a representative $T$ of $(T, 0)$ and a sheaf on $(X, 0) \times T$ inducing $\mathscr{M}$ on $(X \times T, 0)$, (which we also call $\mathscr{M})$ such that for each $t \in T$ the $\mathscr{O}_{(X, 0) \times\{t\}}=\mathscr{O}_{X, 0}$-module $\mathscr{M} \otimes \mathcal{O}_{(X, 0) \times\{t\}}$ is isomorphic to a module of the list. 
Theorem B. Let $R=\mathbb{C}\left\{z_{0}, \ldots, z_{n}\right\} /(f), f \in m-\{0\}, n \geqq 1$. Then the statements (i)(iii) of (1) are equivalent and so are the statements (i)-(iv) of (2).

(1) (i) $f$ defines a simple hypersurface singularity.

(ii) $R$ is of finite CM-representation type.

(iii) Each MCM over $R$ is of finite deformation type.

(2) (i) $f$ defines an $A_{\infty}$ or $D_{\infty}$ singularity.

(ii) $R$ is of countable but not finite deformation type.

(iii) $R$ if of countable but not finite CM-representation type.

(iv) Each MCM over $R$ is of countable deformation type and there exists an MCM over $R$ which is not of finite deformation type.

Remarks. 1) See [Dur] for further characterizations of simple singularities.

2) Since the singularities $A_{\infty}$ and $D_{\infty}$ are not isolated, it follows already from a general theorem of Auslander [Aus2] that they are of infinite $\mathrm{CM}$ representation type. The point is, that these are the only hypersurface singularities which are of countable CM-representation type. We give a complete classification of all MCM's over $A_{\infty}$ and $D_{\infty}$ in $\S 4$.

3) For the simple hypersurface singularities all indecomposable MCM's are explicitly known and their Auslander-Reiten quivers are related to the corresponding Dynkin diagram of type $A_{k}, D_{k}$ or $E_{k}$ respectively (cf. [Knö] for further references). For $A_{\infty}$ and $D_{\infty}$ the Auslander-Reiten quiver of those MCM's which are locally free on a punctured neighbourhood of the singularity are again related to the Dynkin diagrams $A_{\infty}$ and $D_{\infty}$ respectively (cf. [Sch]).

0.4. The modules $M^{ \pm}(\underline{x}, \underline{y})$ which we construct are all of a fixed rank $2^{n-y}$ where $n=\operatorname{dim} R$ and of a fixed minimal number of generators equal to $2^{n}$ (cf. 2.5). There is an estimate, due to $W$. Bruns - and obtained as a consequence of the results in [Bru] - to the effect that any MCM on a hypersurface singularity $R=P /(f)$ without free summands has rank at least $\frac{1}{2}\left(\operatorname{codim}_{R} \operatorname{Sing}(R)-1\right)$ $(\operatorname{Sing}(R)$ denotes the singular locus of $R)$. The actual minimal bound is not known.

We offer the following:

Conjecture A. Any maximal Cohen-Macaulay module $M$ without free summands on an irreducible hypersurface singularity $R$ satisfies:

$$
\operatorname{rank} M \geqq 2^{e}
$$

where $e=\left[\frac{1}{2}\left(\operatorname{codim}_{R} \operatorname{Sing}(R)-2\right)\right]$ and $[q]$ denotes the largest integer $\leqq q$.

Remark that the guess above is stable under taking general hypersurface sections, so that it would be enough to establish it for isolated hypersurface singularities.

\subsection{We give an application to projective geometry:}

Instead of the local case one may consider the graded case. For this replace $P$ by the graded polynomial ring $S=k\left[z_{0}, \ldots, z_{n}\right]$ over an algebraically closed field $k$ and assume that $f \in S$ is homogenous of positive degree. 

$=0$.

Let $X=\operatorname{Proj}_{k}(S /(f)) \subset \mathbb{P}_{k}^{n}$ denote the projective hypersurface defined by $f$

If $n>1$, by a theorem of Grothendieck-Serre (cf. for example [K-L]), graded maximal Cohen-Macaulay modules $M$ over $R=P /(f)$ correspond via sheafification biuniquely to coherent sheaves of $\mathscr{O}_{X}$-modules $\mathscr{A}$ with "no cohomology. in the middle" i.e.

$$
H^{i}(X, \mathscr{M}(v))=0 \quad \text { for } 0<i<n-1=\operatorname{dim} X \text { and } v \in \mathbb{Z} .
$$

Shifting the grading of $M$ by $v$ corresponds to twisting the sheaf $\bar{M}$ by $\left({ }^{\prime \prime r}(v)\right.$. If $X$ is smooth, these sheaves are vector bundles on $X$.

We obtain:

Theorem C. Let $X$ be a projective hypersurface in $\mathrm{IP}_{k}^{n}, n>1, k$ algebraically closed. $X$ is a linear or quadratic hypersurface if and only if there are (up to twist) only finitely many isomorphism classes of indecomposable locally free sheaves $\mathscr{M}$ of $\mathcal{O}_{X}$-modules with vanishing cohomology in the middle:

$$
H^{i}(X, \mathscr{H}(v))=0, \quad v \in \mathbb{Z}, 0<i<n-1 .
$$

In the graded case Conjecture $A$ implies

Conjecture B. Let $X \subset \mathbb{I}_{k}^{n}$ be a smooth hypersurface over an algebraically closed field $k$. Let $\mathscr{M}$ be a vector bundle on $X$ which satisfies

If

$$
H^{i}(X, \mathscr{H}(v))=0, \quad v \in \mathbb{Z}, 0<i<n-1=\operatorname{dim} X .
$$

$$
\operatorname{rank} \mathscr{M}<2^{e}
$$

with $e=\left[\frac{n-2}{2}\right]$, then $\mathscr{M}$ is a direct sum of line bundles

$$
\mathscr{M} \cong \oplus_{i} \mathcal{O}_{X}\left(a_{i}\right)
$$

Remarks. 1) For a linear hypersurface, Conjecture B is true without assumption on the rank by a theorem of Horrocks (cf. [O-S-S]). Since over a regular local ring every MCM is free, this follows also from the Grothendieck-Serre theorem mentioned above.

2) For a quadratic hypersurface, Conjecture B is also true. This follows from the classification of MCM's over the $A_{1}$-singularities in Dimension 1 and 2 and Knörrer's periodicity theorem (cf. also $2.5(4)$ ).

3) The conjecture is at least sharp in all degrees. Look at the Fermat hypersurface $X$ of degree $d$, defined by $f=z_{0}^{d}+\ldots+z_{n}^{d}$. It follows from our construction in $\$ 3$ and sheafification that there are vector bundles on $X$ of rank $2^{e}$ with no cohomology in the middle and which do not contain a line bundle as a direct summand.

\section{Periodic complexes and matrix factorizations}

1.1. We recall the main results about periodic resolutions, maximal CohenMacaulay modules and matrix factorizations as obtained by D. Eisenbud in [Eis, $\$ \$ 4-6]$. These are essential for our construction in the next section. 
A (right-bounded) complex

$$
\mathrm{F} .: \ldots \rightarrow F_{k} \rightarrow F_{k-1} \rightarrow \ldots \rightarrow F_{a} \rightarrow 0, \quad a \in \mathbb{Z},
$$

is called periodic (of period 2) if there is a map of complexes $t: \mathbb{F}$. $\rightarrow \mathbb{F}$. of degree -2 such that $t: F_{i+2} \rightarrow F_{i}$ is an isomorphism for all $i \geqq a$. Identifying $F_{i+2}$ with $F_{i}$ by means of $t$ and setting $G=F_{a}=F_{a+2}=\ldots$ and $F=F_{a+1}=F_{a+3}=\ldots$, we may assume that a periodic complex $\mathbb{F}$. has the form

$$
\text { F.: } \ldots \stackrel{\varphi}{\longrightarrow} F \stackrel{\Phi}{\longrightarrow} G \stackrel{\Psi}{\longrightarrow} F \stackrel{\Phi}{\longrightarrow} G \longrightarrow 0 .
$$

1.2. Special periodic complexes arise from matrix factorizations. Given a ring $P$ and an element $f \in P$, a matrix factorization of $f$ is an ordered pair of morphisms of free $P$-modules, $(\Phi: F \rightarrow G ; \Psi: G \rightarrow F)$, such that

$$
\Phi \circ \Psi=f . \mathrm{id}_{G} \quad \text { and } \quad \Psi \circ \Phi=f . \mathrm{id}_{F} .
$$

If $P$ is local with maximal ideal $m$, the matrix factorization $(\Phi, \Psi)$ is called reduced iff

$$
\operatorname{Im} \Phi \subseteq m_{0} G \text { and } \operatorname{Im} \Psi \subseteq m_{.} F .
$$

Two matrix factorizations $\left(\Phi_{i}: F_{i} \rightarrow G_{i} ; \Psi_{i}: G_{i} \rightarrow F_{i}\right), i=1,2$, of the same element $f \in P$ are equivalent iff there are $P$-linear isomorphisms $\alpha: F_{1} \rightarrow F_{2}, \beta: G_{1} \rightarrow G_{2}$ such that

$$
\beta \Phi_{1}=\Phi_{2} \alpha \quad \text { and } \quad \alpha \Psi_{1}=\Psi_{2} \beta .
$$

If we now set $R=P /(f)$, write ${ }^{-}$for reduction modulo $f$ and denote $\varphi=\bar{\Phi}, \psi$ $=\bar{\Psi}$, then a matrix factorization $(\Phi, \Psi)$ yields a periodic complex of $R$-modules

$$
\mathrm{F}(\Phi, \Psi): \ldots \longrightarrow \bar{F} \stackrel{\varphi}{\longrightarrow} \bar{G} \stackrel{\psi}{\longrightarrow} \bar{F} \stackrel{\varphi}{\longrightarrow} \bar{G} \longrightarrow 0 .
$$

If $f$ is not a zero-divisor on $P$, it follows (cf. [Eis, 5.1]) that $\mathbb{F}(\Phi, \Psi)$ is exact and hence an $R$-free resolution of coker $\varphi$. Furthermore, one has rank $F$ $=\operatorname{rank} G$, $(\mathrm{cf}$. [Eis, 5.4]). Let us also remark (cf. [Eis, 5.5]), that for $f$ a nonzero divisor on $P$ and $(\Phi, \Psi)$ a matrix factorization of $f, \Psi$ is already uniquely determined by $\Phi$.

1.3. Assume now that $R$ is any local Cohen-Macaulay ring and that

$$
\ldots \stackrel{\varphi}{\longrightarrow} G \stackrel{\psi}{\longrightarrow} F \stackrel{\varphi}{\longrightarrow} G \longrightarrow M \longrightarrow 0
$$

is a periodic $R$-free resolution of the $R$-module $M$ (with $F$ and $G$ of finite rank). Then $M$ is a maximal Cohen-Macaulay $R$-module (MCM for short) i.e. depth $M=\operatorname{dim} R$.

This follows from the characterization of depth by Ext's and the obvious fact that $M \cong \operatorname{ker} \varphi$. If $R=P /(f)$ for a non-zero-divisor $f$ in $P$ and if the periodic resolution comes from a matrix-factorization $(\Phi, \Psi)$ of $f$ as above, then $\mathbb{F}(\Phi, \Psi)$ is a minimal resolution of $M=\operatorname{coker} \Phi$ iff $(\Phi, \Psi)$ is reduced. In this case, $M$ has no $R$-free summand (cf. [Eis, $0.1 ; 6.1]$ ). 
1.4. From now on let $P$ denote a regular local ring with maximal ideal $m, f$ a non-zero element of $m$ and $R$ the hypersurface ring $P /(f)$.

The main result on maximal Cohen-Macaulay $R$-modules is that every such module has a periodic free resolution and that all such resolutions arise from matrix factorizations of $f$. More precisely

Theorem ([Eis, 6.1, 6.3]). 1) Every maximal Cohen-Macaulay $R$-module $M$ has a periodic free resolution of the form $\operatorname{IF}(\Phi, \Psi)$ for some matrix factorization $(\Phi, \Psi)$ of $f$. This resolution can be chosen minimal - and hence $(\Phi, \Psi)$ reduced - iff $M$ has no free summand.

2) The associations $(\Phi, \Psi) \mapsto \mathbb{F}(\Phi, \Psi)$ and $(\Phi, \Psi) \mapsto$ Coker $\Phi$ induce bijections between the sets of

(i) equivalence classes of reduced matrix factorizations of $f$ over $P$,

(ii) isomorphism classes of periodic minimal free resolutions over $R$,

(iii) isomorphism classes of maximal Cohen-Macaulay $R$-modules without free summands (the 0-module included).

1.5. Note that the proof of this theorem (as given in [Eis]) is quite elementary. We indicate how the circle closes by repeating the argument which associates to a maximal Cohen-Macaulay $R$-module $M$ a matrix factorization of $f$ :

By the Auslander-Buchsbaum-Serre theorem one has

$$
\text { proj } \operatorname{dim}_{P} M=\operatorname{dim} P-\operatorname{depth} M=1 .
$$

Hence there is a $P$-free resolution of $M$ of length 1 ,

$$
0 \longrightarrow F \stackrel{\Phi}{\longrightarrow} G \longrightarrow M \longrightarrow 0
$$

where $M$ is considered as a $P$-module.

Since $f$ annihilates $M, f \cdot G \subseteq \operatorname{Im} \Phi$ and hence there exists a $P$-linear map $\Psi$ : $G \rightarrow F$ with

$$
\Phi \circ \Psi=f . \mathrm{id}_{G} .
$$

Applying $\Phi$ from the right and noting that $\Phi$ is injective yields then $\Psi \circ \Phi$ $=f$. $\mathrm{id}_{F}$, so that $(\Phi, \Psi)$ is a matrix factorization of $f$ with $\operatorname{Coker} \Phi=M$.

1.6. Now assume that $M$ is a maximal Cohen-Macaulay module over $R$ $=P /(f)$ without free summand. Then, by the theorem above, there exists a reduced matrix factorization $(\Phi, \Psi)$ of $f$ such that $M=\operatorname{Coker} \Phi$.

Keeping the notations from (1.5), $\Phi$ defines a natural map $F \underset{P}{\otimes} G^{*} \rightarrow P$, given by $x \otimes \lambda \rightarrow \lambda(\Phi(x))$ for $x \in F, \lambda \in G^{*}$. The image of this map is an ideal denoted by $I(\Phi)$. Since $(\Phi, \Psi)$ is reduced, it follows that $I(\Phi) \subseteq m$. (Choosing bases for the free $P$-modules $F$ and $G, I(\Phi)$ is just the ideal generated by the entries of the matrix of $\Phi$ with respect to these bases.)

In the same way define $I(\Psi)=\operatorname{Im}\left(G \otimes F^{*} \rightarrow P\right)$. Then we have:

Lemma. With the notations and hypotheses as above, $I(\Phi)$ and $I(\Psi)$ only depend on the isomorphism class of $M=\operatorname{Coker} \Phi$ as an $R$-module. More precisely: 
(i) If $M$ is minimally generated by $\mu$ elements then $I(\Phi)=F_{\mu-1}(M)$,

(ii) $I(\Psi) \cdot \operatorname{det} \Phi=F_{1}(M) \cdot f$,

(iii) $f \in I(\Phi) \cdot I(\Psi) \subseteq(I(\Phi)+I(\Psi))^{2} \subseteq m^{2}$.

Here $F_{a}(-)$ denotes the $a$-th Fitting ideal of a $P$-module, cf. [Kap, App. 43 (b)].

Proof. (i) is essentially the definition of $F_{\mu-1}(M)$, remarking that $\operatorname{Im} \Phi \subseteq m G$ just means $\mu=r k G$.

(ii) (cf. also [Eis, 5.5]): Denote by $\Phi^{\text {ad }}$ the adjoint morphism of $\Phi$,

$$
\Phi^{\mathrm{ad}}=\bigwedge^{r k G-1}(\operatorname{Hom}(\Phi, P)) .
$$

Then one has:

$$
\Phi \circ \Phi^{\mathrm{ad}}=\operatorname{det} \Phi \cdot \mathrm{id}_{G},
$$

(where $\bigwedge^{r k G-1} G$ is identified with $G$ ) and

$$
I\left(\Phi^{\text {ad }}\right)=F_{1}(M)
$$

by the definition of the first Fitting ideal of $M$. From the properties of a matrix factorization one gets

$$
\operatorname{det} \Phi \cdot(\Phi \circ \Psi)=\operatorname{det} \Phi \cdot f \cdot \operatorname{id}_{G}=f \cdot \Phi \circ \Phi^{\text {ad }},
$$

hence, as $\Phi$ is injective,

whence (ii).

$$
\operatorname{det} \Phi \cdot \Psi=f \cdot \Phi^{\text {ad }},
$$

(iii) is a direct consequence of the definitions.

Remark. Although not explicitely required, statement (iii) above shows $f \in m^{2}$. The reason is of course that if $f \in m-m^{2}$, the quotient $R=P /(f)$ is again local regular and all maximal Cohen-Macaulay modules over $R$ are actually free (for which (iii) above yields a new proof).

1.7. To summarize the above, we have associated to every reduced matrix factorization $(\Phi, \Psi)$ an ideal - namely $I(\Phi)+I(\Psi)$ - in $P$, whose square contains $f$.

The key to our results is the following

Proposition. The association $(\Phi, \Psi) \rightarrow I(\Phi)+I(\Psi)$ from (reduced) matrix factorizations of $f$ to ideals of $P$ yields a surjection from the set of isomorphism classes of maximal Cohen-Macaulay modules over $R$ (without free summands) onto the set of those (proper) ideals of $P$ whose square contains $f$.

The proof of this is the content of the next section, see especially (2.4).

As an immediate consequence we get

Corollary. Let $P$ be a regular local ring, $f \in m$ a non-zero element in its maximal ideal and $R=P /(f)$. 
If there are infinitely many different ideals $I \subseteq P$ for which $f \in I^{2}$, then there are infinitely many isomorphism classes of indecomposable maximal Cohen-Macaulay $R$-modules.

This will be exactly the criterion to prove our main results.

Proof of the Corollary. If $M=\oplus M_{i}$ is a decomposition of $M$ into indecomposable summands, a matrix factorization $(\Phi, \Psi)$ for $M$ may be obtained by taking the direct sum of matrix factorizations $\left(\Phi_{i}, \Psi_{i}\right)$ for the $M_{i}$. W. l.o.g. we may assume that no $M_{i}$ is free, hence that $M$ has no free summand. Then, in particular, $I(\Phi)+I(\Psi)=\sum_{i}\left(I\left(\Phi_{i}\right)+I\left(\Psi_{i}\right)\right)$. If there are only finitely many isomorphism classes of indecomposable MCM's over $R$, there are also only finitely many distinct ideals of the form $I(\Phi)+I(\Psi)$ for reduced matrix factorizations $(\Phi, \Psi)$ of $f$. This, together with the proposition, establishes the corollary.

1.8. The constructions and statements above hold similarly in the graded instead of the local case. For this, just replace in 1.4-1.7 the regular local ring $P$ by a graded polynomial ring $S=k\left[z_{0}, \ldots, z_{n}\right]$ over a field $k$ and assume $f$ to be a homogenous element of $S_{+}=\left(z_{0}, \ldots, z_{n}\right) S$ instead of being in $m$.

We leave it to the reader to define graded matrix factorizations and graded resolutions "periodic up to a shift of degrees" and to reformulate (1.4) up to (1.7) in this case.

As mentioned in the introduction, we shall apply the graded case to construct vector bundles on projective hypersurfaces.

\section{Construction of matrix factorizations and MCM's}

2.1. Let $P$ be again an arbitrary commutative ring and $f \neq 0$ an element of $P$. Suppose $f$ is given in the form

$$
f=\sum_{i=1}^{r} x_{i} y_{i}
$$

with $x_{i}, y_{i} \in P$ for $i=1, \ldots, r$. To such a presentation of $f$ we will associate a matrix factorization of $f$ - and hence a maximal Cohen-Macaulay module $M(\underline{x}, \underline{y})$ over $R=P /(f)$ in case $P$ is local regular.

2.2. Let $\mathbb{K}(\underline{x}, P)$ denote the Koszul-complex associated to the sequence $\left(x_{1}, \ldots, x_{r}\right)$ of elements in $P$. Then the underlying graded $P$-module is the exterior algebra $A^{*} P^{r}$, with $P^{r}$ a free $P$-module on the basis $e_{1}, \ldots, e_{r}$. The Koszul-differential will be denoted by $\delta_{-1}$ and is explicitly given by

$$
\left.\delta_{-1}(\omega)=\left(\sum_{i=1}^{r} x_{i} e_{i}\right)\right\lrcorner \omega
$$

for any $\omega \in \Lambda^{*} P^{r}$ where $\lrcorner$ denotes the contraction operator (or "inner product") on $\Lambda^{*} P^{r}$ - (cf. [ALG, §9] for terminology). 
As $f$ is by assumption contained in the ideal generated by the sequence $\left(x_{1}, \ldots, x_{r}\right)$ it annihilates the homology of $\mathbb{K}(\underline{x}, P)$.

But even better, multiplication with $f$ on $\mathbb{K}(x, P)$ is zero-homotopic. A homotopy is given by the following endomorphism of degree +1 on $\mathbb{K}(\underline{x}, P)$,

for any $\omega \in \Lambda^{*} P^{r}$.

$$
\delta_{+1}(\omega)=\left(\sum_{i=1}^{r} y_{i} e_{i}\right) \wedge \omega
$$

Indeed,

$$
\begin{aligned}
\delta_{-1} \circ \delta_{+1}(\omega) & \left.=\left(\Sigma x_{i} e_{i}^{*}\right)\right\lrcorner\left(\Sigma y_{i} e_{i} \wedge \omega\right) \\
& \left.\left.=\left(\left(\Sigma x_{i} e_{i}^{*}\right)\right\lrcorner\left(\Sigma y_{i} e_{i}\right)\right) \wedge \omega-\left(\sum y_{i} e_{i}\right) \wedge\left(\left(\Sigma x_{i} e_{i}^{*}\right)\right\lrcorner \omega\right) \\
& =f \omega-\delta_{+1} \circ \delta_{-1}(\omega) .
\end{aligned}
$$

Since furthermore $\delta_{ \pm 1}^{2}=0$, we have statement (1) of

2.3. Proposition. With the notations as above, set $\delta=\delta_{+1}+\delta_{-1}$.

(1) $\delta$ is an endomorphism of the P-module $\Lambda^{*} P^{r}$ satisfying $\delta^{2}=f \cdot \mathrm{id}_{A^{*} \cdot P^{r}}$. In particular, $(\delta, \delta)$ is a matrix factorization of $f$.

(2) If $P$ is regular local, $f \neq 0$ and $x_{i}, y_{i} \in m$ for all $i$, then:

(i) $(\delta, \delta)$ is reduced and $M(\underline{x}, \underline{y})=\operatorname{coker} \delta$ is a maximal Cohen-Macaulay module over $R=P /(f)$ without free summand.

(ii) $I(\delta)=\left(x_{1}, \ldots, x_{r}, y_{1}, \ldots, y_{r}\right)$.

Remark. It is left as an exercise to the reader to show that $M(\underline{x}, \underline{y})$ is actually free, if one of the $x_{i}$ or $y_{i}$ is a unit.

Proof. Assertion (2.i) follows from (1) by (1.2), (1.3), (ii) follows immediately from the explicit description of $\delta_{ \pm 1}$.

2.4. Proof of Proposition 1.7. Proposition 1.7 is now an easy consequence of Proposition 2.3. Let $I \subset m$ be any ideal with $f \in I^{2}$. Choose a set of generators $\left\{x_{1}, \ldots, x_{r}\right\}$ of $I$. Since $f \in I^{2}$ there exists a presentation $f=\sum_{i=1}^{r} x_{i} y_{i}$ with all $y_{i} \in I$.

Take the reduced matrix factorization $(\Phi, \Psi)=(\delta, \delta)$ of $f$ from 2.3. Then $I(\Phi)$ $+I(\Psi)=I(\delta)$ and by $2.3(2 . \mathrm{ii})$

$$
I(\delta)=\left(x_{1}, \ldots, x_{r}, y_{1}, \ldots, y_{r}\right)=I,
$$

which finishes the proof.

2.5. Remarks. 1) As $\delta_{ \pm 1}$ are graded maps of degree $\pm 1, \delta$ maps $A^{\text {even }} P^{r}$ $=\underset{i \equiv 0(2)}{\bigoplus_{0}} \Lambda^{i} P^{r}$ into $\Lambda^{\text {odd }} P^{r}=\underset{i \equiv y(2)}{\oplus} \Lambda^{i} P^{r}$ and conversely.

In particular, denoting by $\delta^{+}$(resp. $\delta^{-}$) the restriction of $\delta$ to $A^{\text {even }} P^{r}$ (resp. $\left.\Lambda^{\text {odd }} P r\right)$, the matrix factorization $(\delta, \delta)$ of $f$ decomposes as a direct sum

$$
(\delta, \delta)=\left(\delta^{+}, \delta^{-}\right) \oplus\left(\delta^{-}, \delta^{+}\right) .
$$

Accordingly $M(\underline{x}, \underline{y})$ is a direct $\operatorname{sum} M(\underline{x}, \underline{y})=M^{+}(\underline{x}, \underline{y}) \oplus M^{-}(\underline{x}, \underline{y})$ with $M^{ \pm}(\underline{x}, \underline{y})$ $=\operatorname{coker} \delta^{ \pm}$. 
2) Starting from the presentation (2.1) of $f$, one might of course choose another partition of $\left\{x_{1}, \ldots, x_{r}, y_{1}, \ldots, y_{r}\right\}$ into two subsets of $r$ elements such that every index $i=1, \ldots, r$ occurs exactly once in each subset. But it can be seen that the module $M$ obtained from this choice is then isomorphic to $M(\underline{x}, \underline{y})$ - whereas the two summands $M^{+}$and $M^{-}$might get interchanged.

Remark also that $M^{+}$and $M^{-}$are isomorphic to their own duals if $r \equiv 1 \bmod 2$ and isomorphic to the dual of the other if $r \equiv 0 \bmod 2$.

3) If $f$ is irreducible in the UFD $P$, the rank of $M(x, y)$ as an $R$-module is $2^{r-1}$. Namely by [Eis, 5.6] one has $\operatorname{det} \Phi=f^{\mathrm{rank}_{R} \operatorname{coker} \Phi} \cdot \bar{u}(u$ a unit in $P$ ) for every matrix factorization $(\Phi, \Psi)$ of $f$ and by $2.3(1)$

$$
\operatorname{det} \delta \cdot \operatorname{det} \delta=\operatorname{det}\left(f \mathrm{id}_{A \cdot P r}\right)=f^{2 r},
$$

whence $\operatorname{det} \delta= \pm f^{2^{r-1}}$.

It follows easily from this that rank $M^{ \pm}(\underline{x}, \underline{y})=2^{r-2}$ and the reader is encouraged to interpret this result for $r=1$ ! 4) Assume $P=k\left\langle x_{1}, \ldots, x_{r}, y_{1}, \ldots, y_{r}\right\rangle$ and $f=\sum_{i=1}^{r} x_{i} y_{i}$. Then $R=P / f$ is a
singularity of type $A_{1}$ of odd dimension.

If char $k \neq 2$ it can be deduced from the proof of $\mathrm{H}$. Knörrer's periodicity theorem, $[\mathrm{Knö}]$, that indeed the two modules $M^{ \pm}(\underline{x}, \underline{y})$ are non-isomorphic and are the only indecomposable non-free MCM's over $R$. A singularity of type $A_{1}$ of even dimension has exactly one indecomposable non-free MCM. This follows again from the periodicity theorem and the obvious result for $R$ $=k\langle x\rangle / x^{2}$.

\section{Proof of the main results}

3.1. Proposition. Let $P$ be a regular local ring with algebraically closed residue class field $k=P / m, f \in m$ a non zero element and $R=P /(f)$. Suppose

(a) mult $R \geqq 4$ and $\operatorname{dim} R \geqq 1$, or

(b) mult $R \geqq 3$ and $\operatorname{dim} R \geqq 2$.

Then $R$ has infinite CM-representation type.

Proof. By assumption $f \in m^{3}$. Consider the residue $f_{3} \in \mathrm{m}^{3} / \mathrm{m}^{4}$ of $f$ has a homogenous polynomial on $\mathrm{m} / \mathrm{m}^{2}$ and let $C\left(f_{3}\right)$ be the variety defined by $f_{3}=0$ in $\mathbb{P}\left(m / m^{2}\right) \cong \mathbb{P}_{k}^{n} ; n=\operatorname{dim} R$. For every point $\lambda \in C(f)$ we will construct an ideal $I_{\lambda}$ $\subset P$ with $f \in I_{\lambda}^{2}$ as follows:

Choose generators $z_{0}, \ldots, z_{n}$ of $m$ such that in terms of the residue classes $\overline{z_{i}} \in m / m^{2}, \lambda$ is defined by $\overline{z_{1}}=\ldots=\overline{z_{n}}=0$. Then $f \in\left(z_{1}, \ldots, z_{n}\right)+m^{4}$, since $\lambda \in C(f)$.

We define

$$
I_{\lambda}:=\left(z_{1}, \ldots, z_{n}\right)+m^{2}=\left(z_{1}, \ldots, z_{n}, z_{0}^{2}\right) .
$$

Then $I_{\lambda} \neq I_{\mu}$ for $\lambda \neq \mu$ and

$$
I_{\lambda}^{2}=\left(z_{i} z_{j}, 1 \leqq i \leqq j \leqq n, z_{i} \cdot z_{0}^{2}, 1 \leqq i \leqq n, z_{0}^{4}\right)
$$

contains $f$ :

$$
f \in\left[\left(z_{1}, \ldots, z_{n}\right)+m^{4}\right] \cap m^{3}=I_{\lambda}^{2} \cap m^{3} \subset I_{\lambda}^{2} .
$$


By our assumption we have

(a) $C(f)=\mathbb{P}_{k}^{n}$ and $n \geqq 1$, or

(b) $C(f)$ is a cubic hypersurface in $\mathbb{P}_{k}^{n}$ and $n \geqq 2$.

In both cases $\operatorname{dim} C(f) \geqq 1$. So $C(f)$ contains infinitely many points, and there are hence infinitely many different ideals $I$ with $f \in I^{2}$. Now Corollary 1.7 implies the result.

3.2. Proof of Theorem $C$. We replace $P$ by the polynomial ring $S$ $=k\left[z_{0}, \ldots, z_{n}\right], k$ an algebraically closed field, and $f$ by a homogeneous polynomials of positive degree, finally $m$ by $S_{+}=\left(z_{0}, \ldots, z_{n}\right) S$, the irrelevant ideal, and set $R=S /(f)$.

As mentioned in 1.8 , the assertions of $\S 1$ are still true in the graded category. Furthermore, from a presentation

$$
f=\sum_{i=1}^{r} x_{i} y_{i}
$$

by homogeneous elements $x_{i}, y_{i} \in S$, the construction of $\S 2$ gives a graded maximal Cohen-Macaulay module over $R$. Hence, if we choose in the proof of Proposition 3.1 correspondingly homogeneous generators $\left\{z_{1}, \ldots, z_{n}\right\}$ of the homogeneous ideal $S_{+}$, we obtain for $n \geqq 2$ and degree $f \geqq 3$ infinitely many graded MCM's over $R$ which are pairwise not isomorphic, even if we neglect the grading.

By Remark 2.3 and the fact that the ideals $I_{\lambda}$ constructed will contain $S_{+}^{2}$, it follows that the associated modules are actually locally free off $R_{+}=S_{+} /(f)$, the irrelevant ideal of $R$. Sheafification now yields Theorem $C$.

If the degree of $f$ is 1 or 2 , then there are (up to a shift of the grading) only finitely many indecomposable graded MCM's on $R$ (cf. 2.5(4)).

\section{Proof of Theorem A}

3.3. Let $P=k\left\langle z_{0}, \ldots, z_{n}\right\rangle$ denote now a formal or convergent power series ring over an algebraically closed field $k$ of arbitrary characteristic.

If $\operatorname{char}(k) \neq 2$ and

$$
f\left(z_{0}, \ldots, z_{n}\right)=g\left(z_{0}, z_{1}\right)+z_{2}^{2}+\ldots+z_{n}^{2},
$$

where $g$ defines a simple plane curve singularity, $(0.2)$, then $R=P /(f)$ has finite $\mathrm{CM}$-representation type by [Knö]. In order to prove the converse, we establish the existence of infinitely many ideals $I$ with $f \in I^{2}$ for every $f$ which does not decompose into a simple plane $g$ and a sum of squares as above. Then we can apply Corollary 1.7. We proceed by induction on $n=\operatorname{dim} R$.

3.4. Lemma. $f \in k\left\langle z_{0}, z_{1}\right\rangle$ defines a simple curve singularity if and only if for all $x, y \in m$ the following conditions are simultaneously satisfied:

(a) $f \notin(x, y)^{4}$,

(b) $f \notin\left(x^{3}, x^{2} y^{2}, x y^{4}, y^{6}\right)$,

(c) $f \notin\left(x^{2}\right)$. 
Proof. Note that $f \notin\left(x^{2}\right)$ for all $x \in m$ iff $f$ defines a reduced curve singularity. Then applying the method to classify (reduced) simple curve singularities as in [B-P-V, $\$ 11.8$, pp. 61-65] - with modifications as in [K-S] for fields of arbitrary characteristic - gives the lemma. We leave the details to the reader.

3.5. Case $n=1$. If $f$ does not define a simple plane curve singularity, then by the lemma there exist $x, y \in m$ with either
(a) $f \in(x, y)^{4}$, or
(b) $f \in\left(x^{3}, x^{2} y^{2}, x y^{4}, y^{6}\right)$, or
(c) $f \in\left(x^{2}\right)$.

We consider in these cases correspondingly the ideals

(a) $I_{\lambda}=\left(\lambda_{0} x+\lambda_{1} y\right)+(x, y)^{2}, \quad \lambda=\left(\lambda_{0}: \lambda_{1}\right) \in \mathbb{P}_{k}^{1}$,

(b) $I_{\lambda}=\left(x+\lambda y^{2}, y^{3}\right), \quad \lambda \in k$,

(c) $I_{\lambda}=\left(x, y^{\lambda}\right), \quad \lambda \in \mathbb{N}$.

Then $f \in I_{\lambda}^{2}$. This is obvious in Case (a) and (c) and in Case (b) it follows from:

$$
\begin{aligned}
& \left(b_{1}\right) y^{6}=\left(y^{3}\right)^{2} \\
& \left(b_{2}\right) x y^{4}=y\left(x+\lambda y^{2}\right) y^{3}-\lambda y^{6} \\
& \left(b_{3}\right) x^{2} y^{2}=y^{2}\left(x+\lambda y^{2}\right)^{2}-2 \lambda x y^{4}-\lambda^{2} y^{6}
\end{aligned}
$$

and

$$
\left(b_{4}\right) x^{3}=\left(x+\lambda y^{2}\right)^{3}-3 \lambda x^{2} y^{2}-3 \lambda^{2} x y^{4}-\lambda^{3} y^{6} .
$$

For $\lambda \neq \mu$, one has $I_{\lambda} \neq I_{\mu}$ and 1.7 gives infinitely many isomorphism classes of indecomposable MCM's over $R=P /(f)$.

3.6. Case $n>1$. We now assume char $(k) \neq 2$ and proceed by induction on $n$. If mult $R \geqq 3$ then there exist infinitely many ideals $I$ with $f \in I^{2}$ by the proof of 3.1. If mult $R=2$ then we may apply the Weierstraß preparation theorem and Tschirnhausen transformation $(\operatorname{char}(k) \neq 2)$ to obtain

$$
f\left(z_{0}, \ldots, z_{n}\right)=g\left(z_{0}, \ldots, z_{n-1}\right)+z_{n}^{2}
$$

with $g \in k\left\langle z_{0}, \ldots, z_{n-1}\right\rangle$. By the induction hypothesis there are infinitely many pairwise distinct ideals $\tilde{I}_{\lambda} \subset k\left\langle z_{0}, \ldots, z_{n-1}\right\rangle$ with $g \in \tilde{I}_{\lambda}^{2}$. (Notice that this is trivially true if $g=0$.)

The ideals

$$
I_{\lambda}=\tilde{I}_{\lambda} \cdot k\left\langle z_{0}, \ldots, z_{n}\right\rangle+\left(z_{n}\right) \subset k\left\langle z_{0}, \ldots, z_{n}\right\rangle
$$

are pairwise distinct and $f \in I_{\lambda}^{2}$, hence 1.7 gives the result.

\section{Proof of Theorem B}

3.7. From now on let

$$
P=\mathbb{C}\left\{z_{0}, \ldots, z_{n}\right\}
$$

denote the convergent power series ring over the complex numbers. As for the simple singularities in Lemma 3.4 we need a similar characterization of the curve singularities $A_{\infty}$ and $D_{\infty}$. 
Lemma. Let $f \in \mathbb{C}\left\{z_{0}, z_{1}\right\}$ satisfy (a), (b) but not (c) of 3.4. Then there is a coordinate system $x, y \in m$ such that

$$
\begin{aligned}
& A_{\infty_{i}}: f=x^{2}, \quad \text { or } \\
& D_{\infty}: f=x^{2} y .
\end{aligned}
$$

The converse is also true.

Proof. $\mathbb{C}\left\{z_{0}, z_{1}\right\}$ is a unique factorization domain, hence we may write

$$
f=u f_{1}^{e_{1}} \ldots f_{r}^{e_{r}}
$$

with $u$ a unit, $f_{i} \in m$ the irreducible factors of $f$ and $e_{i}$ their multiplicities.

Since (c) is not satisfied, $e_{i} \geqq 2$ for at least one $i$, say $e_{1} \geqq 2$. By (a) mult $(f)<4$, hence $r \leqq 2$ and $\operatorname{mult}\left(f_{i}\right)=1$ for all $i$. Set $x=\sqrt{u} f_{1}$. Then in case $r$ $=1, f=x^{2}$. If $r=2$, set $y=f_{2}$. The residue classes of $x, y$ in $m / m^{2}$ are linearly independent since $f \notin\left(x^{3}\right)+x^{2} m^{2}$ by (b). So $(x, y)=m$ and $f=x^{2} y$. Since the converse is obvious, the lemma follows.

3.8. By the lemma we see that the construction in 3.5 and 3.6 gives countably many isomorphism classes of MCM's for $A_{\infty}$ and $D_{\infty}$. The complete classification of all indecomposable MCM's on $A_{\infty}$ and $D_{\infty}$ in the curve case is given in $\$ 4$. There are only countably many of such modules and by [Knö] this holds in all dimensions. Moreover, in both cases the module presented by the matrix $(x) \oplus(x)$ deforms into each of the modules presented by $\left(\begin{array}{ll}x & y^{k} \\ 0 & x\end{array}\right)$ for $k \geqq 1$, hence it is not of finite deformation type. Again by [Knö] the same holds in higher dimensions.

For the other non simple singularities we constructed in 3.5 and 3.6 families of modules parametrized by $\mathbb{C}$ or $\mathrm{IP}_{\mathbb{C}}^{1}$ and it is easy to see that these families are flat over the parameter-space. Hence none of these modules is of countable deformation type and in particular the singularities are not of countable CMrepresentation type.

Putting everything together, we have almost completed the proof of Theorem B. Using Theorem A, the equivalences in (1) as well as the equivalence of (i), (iii) and (iv) in (2) follow immediately.

Now $A_{\infty}$ deforms into the singularities of type $A_{k}$ and $D_{\infty}$ into the singularities of type $A_{k}$ or $D_{k}$, hence these two singularities are of countable but not finite deformation type.

The converse is essentially contained in Arnold's proof of the classification of simple singularities. A direct argument goes as follows: Let $f$ define a singularity which is neither simple nor $A_{\infty}$ or $D_{\infty}$. Since the deformation theory of $f \in \mathbb{C}\left\{z_{0}, \ldots, z_{n}\right\}$ and $f+z_{n+1}^{2} \in \mathbb{C}\left\{z_{0}, \ldots, z_{n+1}\right\}$ is essentially the same, we may assume that mult $(f) \geqq 3$. If $n$, the dimension of the singularity, is $\geqq 2$ or if $n=1$ and mult $(f) \geqq 4$, the initial part of $f$ defines a projective variety which has moduli, hence the singularity defined by $f$ itself is not of countable deformation type. In case $n=1, \operatorname{mult}(f)=3$, we may assume by Lemma 3.4 
that, in suitable coordinates $x, y$,

$$
f(x, y)=x^{3}+a x^{2} y^{2}+b x y^{4}+c y^{6}
$$

with $a, b, c \in \mathbb{C}\{x, y\}$. Replacing $x^{3}$ by $x\left(x+\lambda y^{2}\right)\left(x+\lambda^{2} y^{2}\right), \lambda \in \mathbb{C}$, gives a family of nonisomorphic singularities (blowing up two times exhibits exactly four points on a rational component of the exceptional divisor, whose cross-ratio varies with $\hat{\lambda}$ ). This completes the proof of Theorem B.

\section{MCM's on $A_{\infty}$ and $D_{\infty}$}

4.1. Let $P$ be a two-dimensional noetherian regular local ring, $m \subset P$ the maximal ideal and $x, y$ generators of $m$. We call a one-dimensional hypersurface ring $R$

$$
\begin{array}{ll}
A_{\infty}, & \text { if } R \cong P /\left(x^{2}\right), \text { or } \\
D_{\infty}, & \text { if } R \cong P /\left(x^{2} y\right) .
\end{array}
$$

Proposition. Let $M$ be an indecomposable MCM over $A_{\infty}$. Then $M$ has a presentation

$$
0 \rightarrow P^{n} \stackrel{\varphi}{\longrightarrow} P^{n} \rightarrow M \rightarrow 0
$$

with $n=1$ or $n=2$ and $\varphi$ one of the following matrices

$$
\begin{aligned}
& n=1: \quad(x),\left(x^{2}\right) \\
& n=2: \quad\left(\begin{array}{rr}
x & y^{k} \\
0 & -x
\end{array}\right), \quad k=1,2,3, \ldots
\end{aligned}
$$

Proof. Consider the short exact sequence

$$
0 \rightarrow \operatorname{ker}(x) \rightarrow M \rightarrow x M \rightarrow 0
$$

where $M$ is any $\mathrm{MCM}$ over $A_{\infty}$. Both, $\operatorname{ker}(x)$ and $x M$ are torsion free $L$ $=P /(x)$-modules. Since $L$ is a discrete valuation ring both modules are free $L$ modules, say

( $x M$ may be 0$)$.

$$
x M \cong L^{m}, \quad \operatorname{ker}(x) \cong L^{n}
$$

By a suitable choice of bases, the natural inclusion $x M \rightarrow \operatorname{ker}(x)$ is given by a diagonal matrix

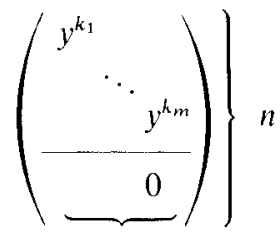

$m$ 
with $0 \leqq k_{1} \leqq \ldots \leqq k_{m} . M$ is an extension of $x M$ by $\operatorname{ker}(x)$ and we obtain a presentation

with

$$
0 \rightarrow P^{n+m} \stackrel{\varphi}{\longrightarrow} P^{n+m} \rightarrow M \rightarrow 0
$$

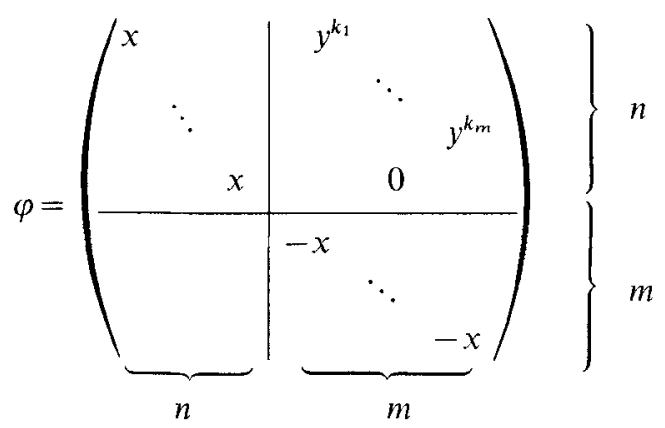

which shows that $M$ is a direct sum of modules of the desired type.

4.2. Proposition. Let $M$ be an indecomposable MCM over $D_{\infty}$. Then $M$ has a representation

$$
0 \rightarrow P^{n} \stackrel{\varphi}{\longrightarrow} P^{n} \rightarrow M \rightarrow 0
$$

with $n=1$ or $n=2$ and $\varphi$ one of the following matrices

$$
\begin{aligned}
n=1: & (x),(y),\left(x^{2}\right),(x y),\left(x^{2} y\right) \\
n=2: & \left(\begin{array}{rr}
x & y^{k} \\
0 & -x
\end{array}\right),\left(\begin{array}{cc}
x y & y^{k+1} \\
0 & -x y
\end{array}\right), \\
& \left(\begin{array}{rr}
x y & y^{k} \\
0 & -x
\end{array}\right),\left(\begin{array}{cc}
x & y^{k+1} \\
0 & -x y
\end{array}\right), \quad k=1,2,3, \ldots
\end{aligned}
$$

Proof. If $M$ is any MCM over $D_{\infty}$, it yields a short exact sequence

$$
0 \rightarrow \operatorname{ker}(y) \rightarrow M \rightarrow y M \rightarrow 0
$$

which exhibits $M$ as an extension of the torsion free (hence MCM) $A_{\infty}=P /\left(x^{2}\right)$ module $y M$ by the free $P /(y)$-module $\operatorname{ker}(y)$. Using the classification of MCM's over $A_{\infty}$ we obtain a presentation

of $M$, where

$$
0 \rightarrow P^{n} \stackrel{\varphi}{\longrightarrow} P^{n} \rightarrow M \rightarrow 0
$$

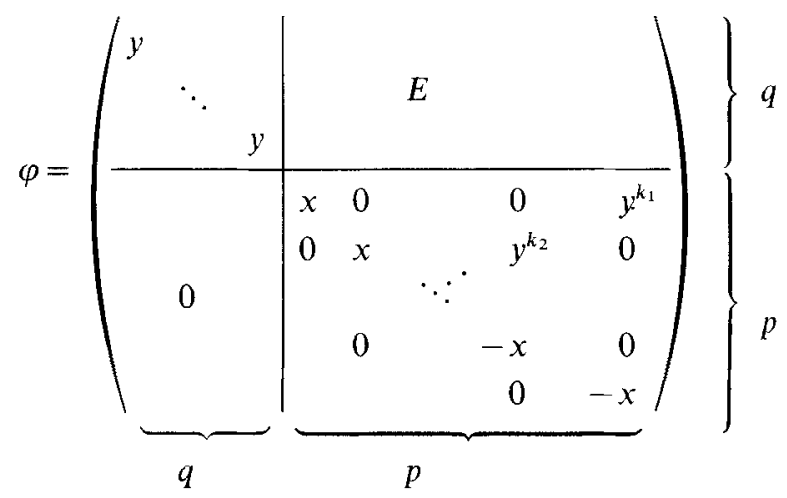


with $0 \leqq k_{1} \leqq \ldots \leqq k_{r} \leqq \infty$, where we put $y^{\infty}:=0, y^{0}:=1$ and use the presentation $\operatorname{coker}\left(\begin{array}{rr}x & 1 \\ 0 & -x\end{array}\right)$ of $\operatorname{coker}\left(x^{2}\right)$. The order of the generators of $y M$ is chosen in such a way that we can apply the Gaußalgorithm to $E$ later on. $q$ is the rank of $\operatorname{ker}(y)$ as $P /(y)$-module, $p=2 r$ (resp. $p=2 r+1$ ) in case the pairity of the summands of $y M$ isomorphic to $(x)$ is even (resp. odd).

Hence

$$
\begin{aligned}
\operatorname{Ext}_{R}^{1}(y M, \operatorname{ker} y) & \cong \operatorname{Ext}_{P}^{1}(y M, \operatorname{ker} y) \\
& \cong(P /(x, y))^{p \cdot q} \quad(\text { as } k=P / m \text {-vectorspaces }) \\
& \cong k^{p \cdot q} .
\end{aligned}
$$

We now use the action of $\operatorname{Aut}(\operatorname{ker}(y)) \times \operatorname{Aut}(y M)$ on $\operatorname{Ext}_{R}^{1}(y M, \operatorname{ker} y)$ via pullback and pushout to simplify $E$ :

Claim. After suitable row and column operations on $\varphi$ the entries of $E$ are either 1 or 0 and no row and column of $E$ contains more than one nonzero entry.

Indeed: The orbits of the action of $G l(q, k) \subset \operatorname{Aut}(\operatorname{ker}(y))$ correspond to a point in the union of Grassmannians $\bigcup G(t, p)$; where $t=\operatorname{rank} E, E$ regarded as a $q \times p$ matrix. $0 \leqq t \leqq \min (p, q)$

Hence we may assume that

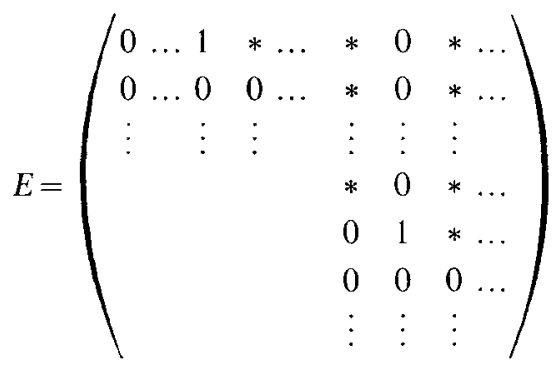

where $*$ indicates an arbitrary entry in $k$.

Now use the action of Aut $(y M)$, i.e. row operations on $E$. For the given order of generators of $y M$, the claim is easily checked, using $k_{1} \leqq k_{2} \leqq \ldots \leqq k_{r}$ and an induction on the cells of the Grassmannians.

Passing now to a minimal presentation, we obtain a direct sum decomposition of $M$ into modules of the desired type. E.g.:

$$
\operatorname{coker}\left(\begin{array}{cc|cc}
y & 0 & 1 & 0 \\
0 & y & 0 & 1 \\
\hline 0 & 0 & x & y^{k} \\
0 & 0 & 0 & -x
\end{array}\right) \cong \operatorname{coker}\left(\begin{array}{cc}
x y & y^{k+1} \\
0 & -x y
\end{array}\right) .
$$




\section{References}

[ALG] Bourbaki, N.: Algèbre, Chap. X. Paris: Hermann 1980

[Arn] Arnol'd, V.I.: Normal forms for functions near degenerate critical points, the Weyl groups of $A_{k}, D_{k}, E_{k}$ and Lagrangian singularities. Funkts. Anal. 6, 254-272 (1972)

[A-V] Artin, M., Verdier, J.L.: Reflexive modules over rational double points. Math. Ann. 270, 79-82(1985)

[Aus1] Auslander, M.: Rational singularities and almost split sequences. Trans. Am. Math. Soc. 293, 511-531 (1986)

[Aus2] Auslander, M.: Isolated singularities and almost split sequences. Ottawa Proceedings 1984, SLN

[B-P-V] Barth, W., Peters, C., van de Ven, A.: Compact complex surfaces. Erg. d. Math. Band 4, Berlin-Heidelberg-New York-Tokio: Springer 1984

[Bru] Bruns, W.: The Eisenbud-Evans generalized principal ideal theorem and determinantal ideals. Proc. Am. Math. Soc. 83, 19-26 (1981)

[Dur] Durfee, A.: Fifteen characterizations of rational double points and simple critical points. L'Enseignement Math. 25, 131-163 (1979)

[Eis] Eisenbud, D.: Homological Algebra on a complete intersection, with an application to group representations. Trans. Am. Math. Soc. 260, 35-64 (1980)

[Esn] Esnault, H.: Reflexive modules on quotient singularities. J. Reine Angew. Math. (in press) (1987)

[G-K] Greuel, G.-M., Knörrer, H.: Einfache Kurvensingularitäten und torsionsfreie Moduln. Math. Ann. 270, 417-425 (1985)

[Kap] Kaplansky, 1.: Commutative Rings, Rev. Edition, Univ. of Chicago Press, Chicago, 1974

[K-S] Kiyek, K., Steinke, G.: Einfache Kurvensingularitäten in beliebiger Charakteristik. Arch. Math. (in press) (1987)

[K-L] Kleiman, S.L., Landolfi, J.: Geometry and deformation of special Schubert varieties. In: Oort, F. (ed.), Algebraic geometry, Oslo 1970, pp. 97-124. Wolters-Noordhoff Publ. Groningen 1972

[Knö] Knörrer, H.: Cohen-Macaulay modules on hypersurface singularities I. Invent. Math. 88, 153-164 (1987)

[O-S-S] Okonek, C.. Schneider, M., Spindler, H.: Vectorbundles on complex projective spaces. Boston-Basel-Stuttgart: Birkhäuser 1980

[Sch] Schreyer, F.-O.: Finite and countable CM-representation type. To appear in Proc. Symp. on Singularities, Representations of algebra and vector bundles

[Sie] Siersma, D.: Isolated line singularities. In: Singularities. Proc. Symp. Pure Math. 40, 485$496(1983)$ 